\title{
Avaliação de efeitos Não Lineares em Sistemas OFDM pela Figura de Mérito EVM
}

\author{
Jair A. L. Silva, Bernardo L. Valentim e Marcelo E. V. Segatto
}

\begin{abstract}
Resumo-Este trabalho apresenta uma análise de desempenho, via vetor de erro de magnitude (EVM), de sinais gerados por sistemas de multiplexação por divisão de freqüências ortogonais (OFDM) com efeitos não lineares provenientes de amplificadores de potência (PA) e ceifamento de pico em canais com ruído gaussiano branco (AWGN). A ineficiência do clipping em reduzir a razão potência de pico e potência média (PAPR) de sinais OFDM é confirmado pelos resultados de simulações computacionais. Além disso, é demonstrada a capacidade da figura de mérito EVM em estimar e corrigir desvios de fase provocados por desbalanceamento $\mathrm{I} / \mathrm{Q}$ de sinais multiportadoras.
\end{abstract}

Palavras-Chave-Multiplexação por divisão de frequências ortogonais, vetor de erro de magnitude, amplificadores de potência, ceifamento de pico, desbalanceamento $I / Q$.

Abstract-This paper presents a performance analysis, through error vector magnitude (EVM), of orthogonal frequency division multiplexing (OFDM) signals corrupted by power amplifiers (PA) and clipping non-linear distortions in additive white gaussian noise (AWGN) channels. The simulation results confirms the non affectivity of clipping process as a PAPR reduction technique, and also shows the capacity of EVM figure of merit in estimation and correction of phase deviation in OFDM signals generated by $\mathrm{I} / \mathrm{Q}$ imbalance simulation.

Keywords-Orthogonal frequency division multiplexing (OFDM), error vector magnitude (EVM), power amplifier (PA), clipping, I/Q imbalance.

\section{INTRODUÇÃO}

Como padrão de transmissão nas tecnologias de radiodifusão digital de audio e vídeo, redes locais sem fio, IEEE 802.16 (WiMAX), entre outros, a modulação OFDM é preconizado como solução efetiva à interferência intersimbólica em canais dispersivos [1]. A tolerância ao espalhamento por atraso multipercurso, eficiência espectral, combate à ICI (inter-channel interference) e simplicidade na equalização, são algumas das vantagens inerentes ao uso desta técnica para a transmissão de dados a altas taxas de transferência [2], [3]. Entretanto, como modulação multiportadora, a técnica OFDM gera sinais com larga faixa de excursão, caracterizado por altos valores da razão potência de pico e potência média do sinal, PAPR (peak to average power ratio). Isto compromete o desempenho do sistema pela inserção de distorções não lineares por dispositivos como os amplificadores de potência normalmente projetados para operarem perto da região de saturação para alto rendimento [1], [4]. A avaliação de tais distorções é melhor realizada utilizando-se da métrica EVM

Laboratório de Telecomunicações - http://www.labtel.ele.ufes.br Universidade Federal do Espírito Santo, Vitória, e-mail: segatto@ele.ufes.br Trabalho parcialmente fianciado pelo CNPq, projeto número 309049/2006-7. (error vector magnitude), que além de expressar a qualidade da recepção de sistemas de modulação digital, provê uma simples e quantitativa figura de mérito de sinais modulados digitalmente, pelo fato de representar a diferença entre os vetores de símbolos transmitidos e recebidos de uma determinada diagrama de constelação [5]. Além disso, a EVM extraí informações da diferença de fase entre os símbolos complexos gerados e medidos, o que permite uma melhor caracterização dos efeitos físicos de um canal de comunicação. A sua capacidade em identificar as distorções provocadas por não linearidades, ruído de fase, entre outros, possibilitou a sua especificação como figura de mérito dos padrões IEEE 802.11a-1999 e WCDMA (wideband code division access) [6], [7].

O presente artigo tem por objetivo principal unificar os dispersos conceitos da figura de mérito EVM encontrados na literatura visando uma analise via simulação computacional dos efeitos não lineares provocados por amplificadores de potência e pelo ceifamento de pico ou clipping em sistemas OFDM. Além disso, para ilustrar a capacidade da métrica em estimar e corrigir erros de fase, analisa-se também o efeito do desbalanceamento I/Q em sinais gerados por tais sistemas multiportadoras.

Este artigo encontra-se organizado da seguinte maneira. Realiza-se na seção II uma explanação dos conceitos básicos que abrangem a técnica de multiplexação OFDM, bem como dos efeitos causados em sinais multiportadoras por PA, clipping e desbalanceamento I/Q. A unificação de conceitos referentes à figura de mérito EVM é realizada na seção III, com especial ênfase atribuída às relações entre esta métrica, a taxa de erro de bits BER (bit error rate), a razão potência de sinal e potência de ruído SNR (signal to noise ratio), e a taxa de erro de modulação MER (modulation error rate). A análise dos resultados de simulação e as conclusões pertinentes são realizadas nas seções seguintes.

\section{OFDM (Orthogonal Frequency Division Multiplexing)}

A comunicação OFDM tem como princípio básico a transmissão paralela de informações em várias subportadoras ortogonais espectralmente sobrepostas. Para isso, a técnica divide o espectro de freqüências do canal de transmissão em $N_{s}$ subcanais, de tal forma que a duração $T_{s}$ de um símbolo OFDM seja maior que o retardo ou atraso de propagação do canal $\tau_{\max }$, minimizando assim os efeitos da interferência entre símbolos subseqüentes, ISI (inter-symbol interference). Para eliminar os efeitos da interferência entre subcanais, aplica-se uma extensão cíclica no símbolo em um intervalo de 
guarda, o qual simplifica o processo de equalização do canal. A Figura 1, mostra a configuração básica de um sistema de transmissão digital OFDM. A sequiência de dados digitais de entrada é dividida em $N$ subsequencias através do conversor serial para paralelo de $m$ bits, sendo cada uma mapeada em um subsímbolo complexo $X=X_{i}+j X_{Q}$ em uma constelação de $M=2^{m}$ níveis. Após preenchimento com $N_{z}=N_{s}-N$ zeros pelo bloco zero padding, efetua-se a multiplexação e/ou a modulação digital com a transformada inversa de Fourier, IFFT (inverse fast Fourier transform) em um conjunto de $L=2 N_{s}+2$ subsímbolos, $X_{k}=$ $\left[0, X_{0}, X_{1}, \cdots, X_{N_{s}-1}, 0, X_{N_{s}-1}^{*}, \ldots, X_{1}^{*}, X_{0}^{*}\right]^{T}$ com simetria hermitiana para a geração de um símbolo OFDM com coeficientes reais, onde $*$ representa o complexo conjugado. Após conversão paralelo para serial, ao sinal OFDM no domínio do tempo é aplicado a extensão cíclica pelo bloco prefixo cíclico, CP (cyclic prefix) obtendo-se finalmente a seqüência $x_{n}=\left[x_{L-G}, \cdots, x_{N-1}, x_{0}, x_{1}, \cdots, x_{L-1}\right]$, para $G$ a quantidade de amostras do intervalo de guarda. A remoção do $\mathrm{CP}$, a demodulação via FFT (fast Fourier transform), remoção da simetria hermitiana e das subportadoras inativas (zero padding) e o demapeamento fazem parte da recepção do sinal OFDM transmitido. Salienta-se que operações adicionais relativos à equalização de canal e sincronismo são por conveniência aqui omitidos.

Matematicamente, a forma de onda de um símbolo OFDM de tempo contínuo é dado por

$$
x_{n}=\sum_{k=0}^{L-1} X_{k} \cdot e^{j 2 \pi f_{k} t} \quad-T_{g} \leq t<T_{s},
$$

onde $\left\{X_{k}\right\}_{k=0}^{L-1} \quad$ são os símbolos complexos, $\left\{\exp \left(j 2 \pi f_{k} t\right)\right\}_{k=0}^{L-1}$ as subportadoras, $f_{k}=k / T_{s}$ a frequência da subportadora $k$ e $T_{g}$ a duração do intervalo de guarda.

\section{A. Amplificadores de Potência}

Essenciais em sistemas de comunicação de dados, os amplificadores de potência PA, introduzem distorção dentro e fora do espectro de potência do sinal de transmissão, devido à não linearidades inerentes aos mesmos. A degradação do desempenho em sistemas OFDM é ainda maior devido aos altos valores de PAPR dos sinais gerados. Um dos modelos de PA mais empregados em tais sistemas é o SSPA (solid-state power amplifier) no qual assume-se resposta em frequência não seletiva, simplificação importante devido à dificuldade da modelagem de amplificadores não lineares [1]. Considerandose como entrada do PA o sinal $x_{n}(t)=A(t) e^{j \phi(t)}$, o sinal na sua saída será

$$
y_{n}=G[A(t)] \cdot e^{j\{\phi(t)+\Phi[A(t)]\}}
$$

onde, para o modelo SSPA, $\Phi(A)=0$ representa a conversão modulação de amplitude para modulação de fase AM/PM (amplitude modulation to phase modulation) do PA e

$$
G(A)=\frac{g_{0} A}{\left[1+\left(A / A_{\text {sat }}\right)^{2 p_{\text {sat }}}\right]^{1 / 2 p_{\text {sat }}}},
$$

a característica AM/AM (amplitude modulation to amplitude modulation), para $g_{0}$ o ganho de amplitude, $A_{\text {sat }}$ o nível de saturação de entrada e $p_{\text {sat }}$ o fator que controla a curvatura da região de saturação do PA.

\section{B. Ceifamento de Pico}

O clipping é a mais simples e por isso mais utilizada técnica para diminuir o PAPR dos sinais OFDM e consequentemente as distorções inseridas pela PA. Após o ceifamento, o sinal pode ser descrito no domínio do tempo como

$$
y_{n}=\left\{\begin{array}{cc}
x_{n} & \left|x_{n}\right| \leq A_{\max } \\
A_{\max } e^{j \angle x_{n}} & x_{n}>A_{\max }
\end{array}\right.
$$

onde $x_{n}$ é o sinal gerado e $A_{\max }$ o limiar de clipping. Garante-se com isso que a amplitude do sinal não exceda $A_{\max }$ enquanto preserva-se a fase do sinal gerado. Devese no entanto, controlar o nível de ceifamento, uma vez que degradações severas no desempenho do sistema são dele dependentes.

\section{Desbalanceamento $I / Q$}

A conversão direta de um sinal na faixa de rádio frequência, RF (radio frequency), para banda base no processo de recepção pode provocar um desbalancemento no ganho/fase (I/Q) do próprio sinal. Em sinais OFDM isso acarreta em interferências entre as subportadoras, e portanto, em distorções que acontecem dentro da faixa de interesse do sinal. Matematicamente, este desbalanceamento é caracterizado pelos parâmetros $\epsilon$ e $\Delta_{\phi}$, os quais representam o desbalamceamento em ganho e em fase respectivamente. O modelo de sinal em banda base é então definido como

$$
y_{n}=\alpha \cdot x_{n}+\beta x_{n}^{*},
$$

onde $\alpha=\cos \left(\Delta_{\phi}\right)+j \cdot \epsilon \cdot \operatorname{seno}\left(\Delta_{\phi}\right)$ e $\beta=\epsilon \cdot \cos \left(\Delta_{\phi}\right)-$ $j \operatorname{seno}\left(\Delta_{\phi}\right)$. Para o caso em que pretende-se simular apenas o desbalanceamento de fase faz-se $\epsilon=0$, consequentemente $\alpha=\cos \left(\Delta_{\phi}\right)$ e $\beta=-j \operatorname{seno}\left(\Delta_{\phi}\right)$.

\section{EVM (Error Vector Magnitude)}

Para um melhor entendimento do procedimento de medida da EVM, considere o quadrante de um diagrama de constelação da Figura 2, onde são apresentados o vetor de referência $X_{k}$ relativo a um dos possíveis símbolos transmitidos (sinal ideal) de coordenadas $X_{I}=1$ e $X_{Q}=j$, para $j=\sqrt{-1}$, o vetor medido $Y_{k}$ que ilustra a trajetória de um símbolo recebido (sinal medido) de coordenadas $\left(Y_{I}, Y_{Q}\right)$, e o vetor de erro $D_{k}=Y_{k}-X_{k}$, o qual representa a distancia Euclidiana entre tais sinais. Sendo cada ponto do plano I (In phase) versus Q (Quadrature) a especificação de um dos $2^{m}$ possíveis símbolos gerados pela combinação de $m$ bits, qualquer desvio neste provocado por ruído ou distorção, linear ou não, cria uma distancia escalar entre os referidos fasores cuja magnitude de erro $E_{k}=\left|Y_{k}\right|-\left|X_{k}\right|$ e o erro de fase $\phi_{k}=\angle\left(Y_{k}\right)-\angle\left(X_{k}\right)$ são perfeitamente mensuráveis pela métrica EVM [9].

Portanto, como distância escalar - magnitude do vetor diferença - entre dois fasores, a EVM pode ser definido como o valor RMS (root-mean-square) da diferença entre um conjunto de símbolos medidos e um conjunto de símbolos transmitidos, matematicamente expresso pela relação,

$$
E V M=\left[\frac{\frac{1}{N} \sum_{k=0}^{N-1}\left|Y_{k}-X_{k}\right|^{2}}{\frac{1}{N} \sum_{i=0}^{N-1}\left|X_{k}\right|^{2}}\right]^{\frac{1}{2}}
$$




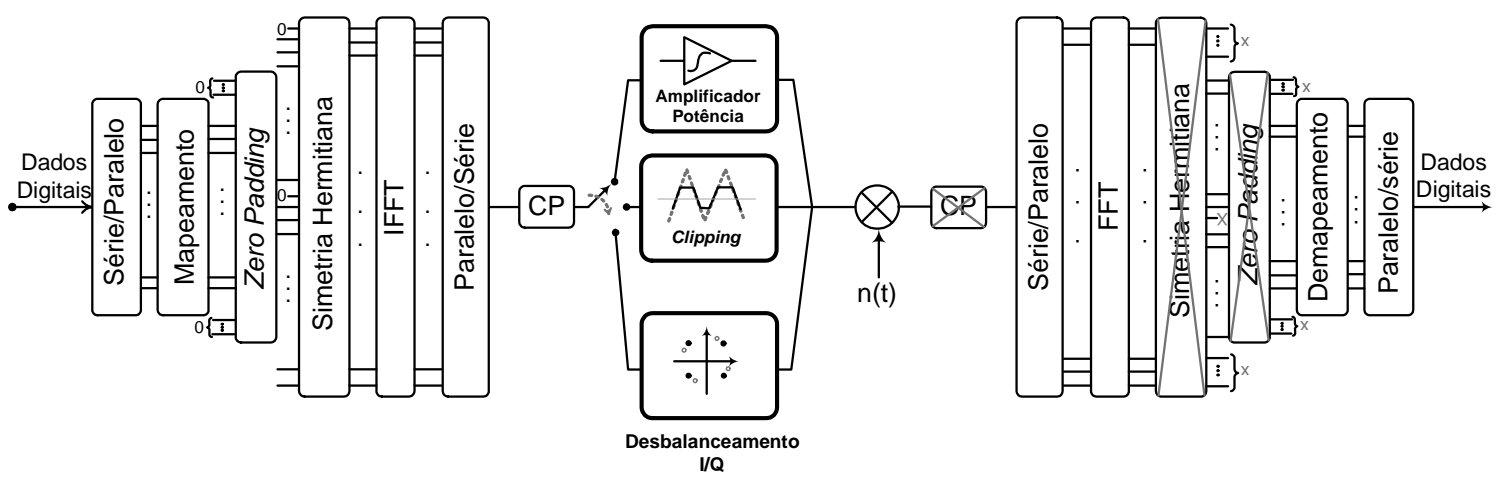

Fig. 1. Configuração básica de um sistema OFDM, com efeitos não lineares gerados por amplificadores de potência e clipping e ruído de fase inserido pelo bloco desbalanceamento I/Q. Sincronismo, estimação e equalização de canal são por conveniência omitidos.

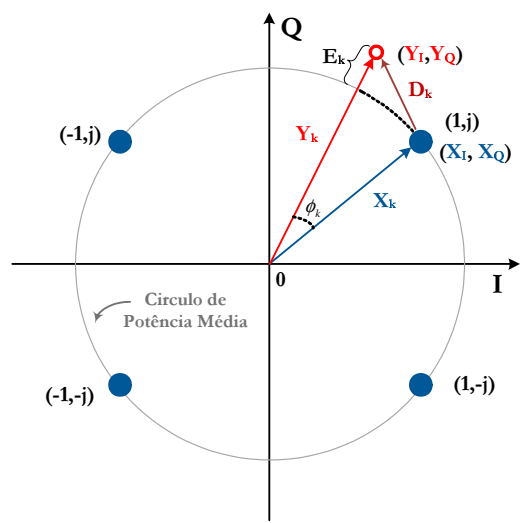

Fig. 2. Diagrama de constelação 4-QAM ilustrativo.

onde $N$ representa a quantidade de símbolos transmitidos/recebidos, $X_{k}=X_{I}+j X_{Q}$ e $Y_{k}=Y_{I}+j Y_{Q}$ os símbolos complexos ideais e medidos respectivamente [5], [8]. Entretanto, é comum encontrar na literatura a definição matemática da EVM conforme

$$
E V M=\sqrt{\frac{\frac{1}{N} \sum_{k=0}^{N-1}\left|D_{k}\right|^{2}}{X_{\max }^{2}}},
$$

para $\left|D_{k}\right|^{2}=\left|Y_{k}-X_{k}\right|^{2}$ o vetor erro de sinal e $X_{\max }$ o ponto da constelação de maior amplitude. Esta designação detém a vantagem de facilitar e/ou diminuir a implementação computacional da citada métrica [7].

\section{A. Diagramas de Constelação Normalizadas}

Normalmente, sistemas de transmissão multiportadora utilizam portadoras piloto para reconhecimento e estimação do canal de comunicação, os quais empregam a modulação digital BPSK (binary phase shift keying) para diferenciarem-se das portadoras de dados. Nestes casos, o cálculo eficiente da EVM requer a normalização dos diagramas de constelação, de tal forma que o valor médio quadrático da amplitude de todos os possíveis símbolos da constelação seja unitário. Deve-se portanto, normalizar tanto a constelação transmitida quanto a recebida para uma justa comparação entre o conjunto de valores inteiros com os valores de tensão arbitrários medidos. Para tal, divide-se a potência de cada símbolo medido, $P_{Y_{k}}$ pela potência média do total destes, $P_{Y_{m e d}}$, obtendo-se $P_{Y_{k m e d}}=\frac{P_{Y_{k}}}{P_{Y_{m e d}}}$, sendo

$$
P_{Y_{\text {med }}}=\frac{\frac{1}{N} \sum_{k=0}^{N-1}\left[\left(Y_{I}\right)^{2}+\left(Y_{Q}\right)^{2}\right]}{N},
$$

para $Y_{I}$ e $Y_{Q}$ os componentes em fase e quadratura dos níveis de tensão dos $N$ símbolos recebidos. Da mesma maneira, obtém-se $P_{X_{k m e d}}=\frac{P_{X_{k}}}{P_{X_{m e d}}}$, onde

$$
P_{X_{\text {med }}}=\frac{\sum_{p=1}^{m}\left[\sum_{q=1}^{m}(2 p-1-m)^{2}+(2 q-1-m)^{2}\right]}{M}
$$

é a amplitude média quadrática normalizada dos $M=2^{m}$ possíveis símbolos da constelação de $m$ níveis, $1 \leq p \geq m$ e $1 \leq q \geq m$. Com isso, o valor RMS da EVM torna-se

$$
E V M=\left[\frac{\frac{1}{N} \sum_{k=0}^{N-1}\left|Y_{I_{n}}-X_{I_{n}}\right|^{2}+\left|Y_{Q_{n}}-X_{Q_{n}}\right|^{2}}{P_{X_{m e d}}}\right]^{\frac{1}{2}},
$$

onde, $Y_{I_{n}}=Y_{I} \cdot\left|A_{m e}\right|, X_{I_{n}}=X_{I} \cdot\left|A_{m e}\right|, Y_{Q_{n}}=Y_{Q} \cdot\left|A_{m e}\right|$, $X_{Q_{n}}=X_{Q} \cdot\left|A_{m e}\right|$, para $A_{m e}=\sqrt{\frac{N}{P_{Y_{m e d}}}}$ e $A_{i d}=\sqrt{\frac{M}{P_{X_{m e d}}}}$ os fatores de normalização dos símbolos medidos e símbolos únicos, respectivamente. As curvas da Figura 3; obtidas via simulação computacional da transmissão de $N_{O F D M}=100$ sinais OFDM de $N=1024$ subportadoras em um canal com ruído AWGN para diversos valores de SNR; ilustram a independência da EVM $\left(20 \times \log _{10}(E V M)\right)$ da modulação empregada nas subportadoras depois da normalização.

Ressalta-se que após a normalização, o denominador da equação 9 é igual a 1 . Além disso assume-se que a probabilidade de ocorrência de cada símbolo único do diagrama de constelação é a mesma para todos os símbolos transmitidos.

No padrão IEEE $802.11 \mathrm{a} / \mathrm{g}$, em que $L_{f} \geq 16$ frames de $N_{O F D M} \geq 20$ sinais OFDM contendo $N=52$ subportadoras, a EVM pode, a partir de (9), ser simplificada para

$$
E V M \approx \sqrt{\frac{\sum_{l=0}^{L_{f}-1} \sum_{k=0}^{N-1}\left|Y_{I_{n}}-X_{I_{n}}\right|^{2}+\left|Y_{Q_{n}}-X_{Q_{n}}\right|^{2}}{P_{0} L_{f} N}},
$$

onde $Y_{I_{n}}=Y_{I_{k, l}}\left|A_{m e}\right|, X_{I_{n}}=X_{I_{k, l}}\left|A_{m e}\right|, Y_{Q_{n}}=$ $Y_{Q_{k, l}}\left|A_{m e}\right|, X_{Q_{n}}=X_{Q_{k, l}}\left|A_{m e}\right|$, considerando-se que o valor 


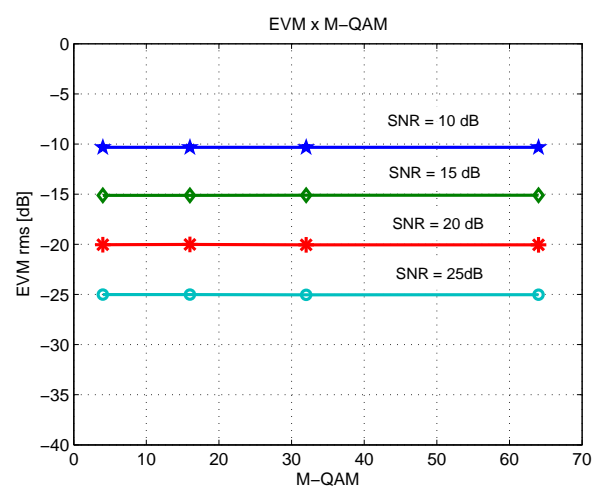

Fig. 3. EVM para modulações $(M=4,16,32,64)$-QAM em canais com ruído aditivo gaussiano branco AWGN, para diversos valores de SNR.

da EVM é o mesmo em cada frame e $P_{0}=\frac{1}{L} \sum_{l=1}^{L_{f}} P_{X_{m e d}}$ é a potência média da constelação usada. [9].

\section{B. Relações Matemáticas entre SNR, EVM, e BER}

Análise de desempenho computacional nos termos taxa de erro de bits BER, e relação potência de sinal e potência de ruído SNR, é por motivos de simplicidade uma das mais utilizadas em sistemas de comunicação. A medição direta da potência do sinal sobre a potência do ruído permite predizer a quantidade de bits errados ou não no processo de decisão do receptor [5]. Existem tabuladas na literatura enumeras relações probabilísticas que numericamente descrevem o comportamento de sinais com modulação digital em canais com ruído gaussiano. Para tanto, considere a transmissão em canais AWGN de sinais uni/multiportadoras, com modulação MQAM de sequência de bits codificados com código Gray, e com detecção coerente. A probabilidade de erro de bits, ou taxa de erro de bits é dada por

$$
B E R=\frac{2\left(1-\frac{1}{\sqrt{M}}\right)}{\log _{2}(M)} \cdot \operatorname{erfc}\left(\sqrt{\frac{3 S N R}{(2 M-2)}}\right),
$$

onde $\operatorname{erfc}(x)=\frac{2}{\sqrt{\pi}} \int_{x}^{\infty} e^{-y^{2}} d t$ é a função erro complementar, M é o tipo de modulação empregada (para 16-QAM, $\mathrm{M}=16$ ) e a SNR matematicamente descrita pela razão

$$
S N R=\frac{\frac{1}{N} \sum_{k=1}^{N}\left[\left(X_{I}\right)^{2}+\left(X_{Q}\right)^{2}\right]}{\frac{1}{N} \sum_{k=1}^{N}\left[\left(n_{I}\right)^{2}+\left(n_{Q}\right)^{2}\right]},
$$

para $X_{I}$ e $X_{Q}$ os componentes em fase e quadratura do sinal transmitido, $n_{I}$ e $n_{Q}$ as amplitudes em fase e quadratura do ruído gaussiano de densidade espectral de potência igual a $\frac{N_{0}}{2}$ [5]. Em sistemas amostrados à taxa de símbolos, a SNR pode ser definido por

$$
S N R=\frac{E_{s}}{N_{0}}=\frac{\log _{2}(M) E_{b}}{N_{0}},
$$

onde $E_{s}=\log _{2}(M) E_{b}$ é a energia de cada símbolo constelação e $E_{b}$ a energia de cada bit que o compõem. É possível afirmar, com atento à equação (10), que a EVM é essencialmente o erro de magnitude normalizada entre a constelação medida e a constelação gerada. Em um canal com ruído gaussiano branco, a relação entre a SNR e a EVM, ilustrada na Figura 3, é evidenciada por

$$
E V M \approx\left[\frac{1}{S N R}\right]^{\frac{1}{2}} \approx\left[\frac{N_{0}}{E_{s}}\right]^{\frac{1}{2}}
$$

que em dB torna-se $E V M \approx-20 \times \log _{10}(S N R)$ [5]. Assim, sabendo que $S N R \approx 1 /\left(E V M^{2}\right)$ reescreve-se (11) como

$$
B E R=\frac{2\left(1-\frac{1}{\sqrt{M}}\right)}{\log _{2}(M)} \cdot \operatorname{erfc}\left(\sqrt{\frac{3}{E V M^{2} \cdot(2 M-2)}}\right),
$$

como relação matemática entre a BER e a EVM medida diretamente de diagramas de constelações quadradas M-QAM. A Figura 4 mostra tais relações obtidas através da simulação de um sistema OFDM de $N_{O F D M}=100$ sinais de $N=1024$ subportadoras moduladas em 16-QAM, em um canal AWGN para variados valores de SNR.
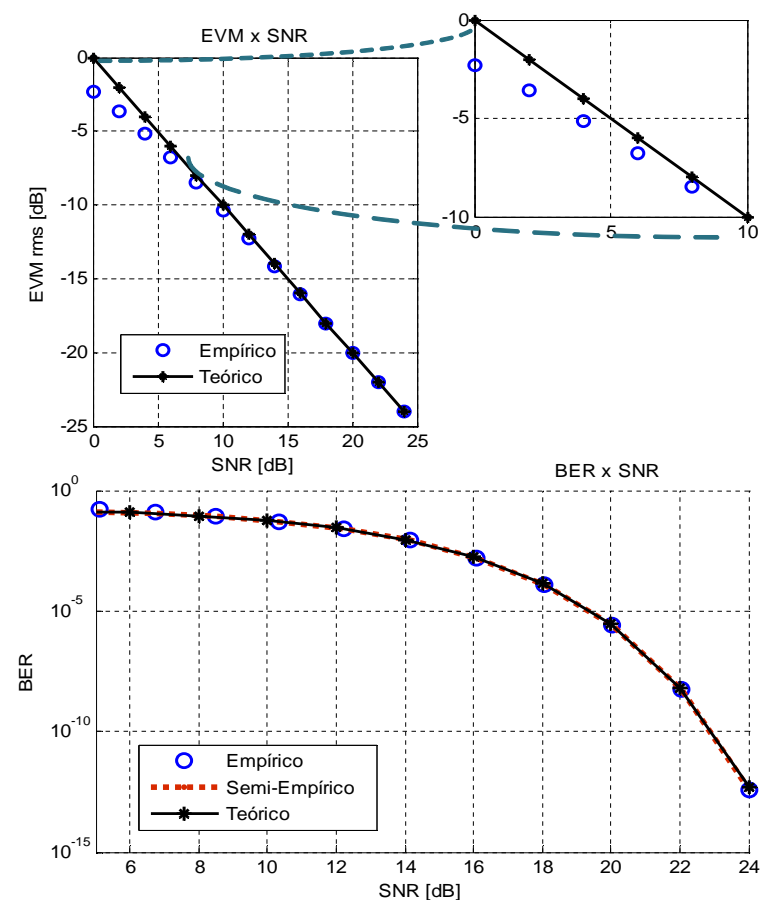

Fig. 4. EVM e BER versus SNR de um sistema OFDM com mapeamento 16-QAM em canal AWGN.

A relação empírica entre a EVM e a SNR refere-se ao resultado da simulação, ou seja, à EVM obtida via simulação computacional aplicada à relação (14). O traço referente ao EVM versus SNR Teórico consiste em aplicar os valores de SNR dados como entrada na equação (14). O comportamento empírico do gráfico de desempenho BER versus SNR provém da simulação do sistema OFDM parametrizado conforme descrito anteriormente. Os traços Teórico e Semi-Empírico são provenientes do uso das equações (11) e (15) respectivamente. Observa-se uma diferença de aproximadamente $1.5 \mathrm{~dB}$ na comparação entre o EVM por SNR Empírico (simulado) e o Teórico para a $S N R=2 \mathrm{~dB}$. Uma ponderação sobre essa pequena discrepância sugere a delimitação de um valor a partir do qual emprega-se a relação (14). Deve-se no entanto realçar a exatidão das curvas de desempenho, exaltando a gama 
de valores acima de $10^{-6}$ de real interesse em sistemas de telecomunicações.

No entanto, em sistemas em que não se conhece a estatística e a proveniência das interferências dominantes, torna-se apropriada a definição da grandeza taxa de erro de modulação MER (modulation error rate) conforme

$$
M E R=E V M_{d B}=-20 \log _{10}(E V M)+10 \log _{10}\left(\frac{\left\langle V^{2}\right\rangle}{V_{\max }^{2}}\right)
$$

onde $\left\langle V^{2}\right\rangle$ é o valor quadrático médio da tensão de todos os pontos da constelação e $V_{\max }$ a tensão do ponto da constelação de maior amplitude [10]. Em (16), EVM= $r_{e} / V_{\text {max }}$, para $r_{e}$ a magnitude do raio do vetor do dados recebidos determinada pelo valor médio quadrático do desvio entre os dados recebidos e os pontos da constelação. Tornase, portanto, desnecessária a normalização do diagrama de constelação, desde que os tons pilotos; em casos em que estes são utilizados; sejam retirados antes da avaliação da métrica. Como valor médio da SNR de toda a constelação, a MER pode assim ser aplicada na equação (15) para a determinação aproximada de uma taxa de erro de bits referencial. A Figura 5 mostra a avaliação de desempenho via simulação do referido sistema OFDM com a figura de mérito da equação (16).

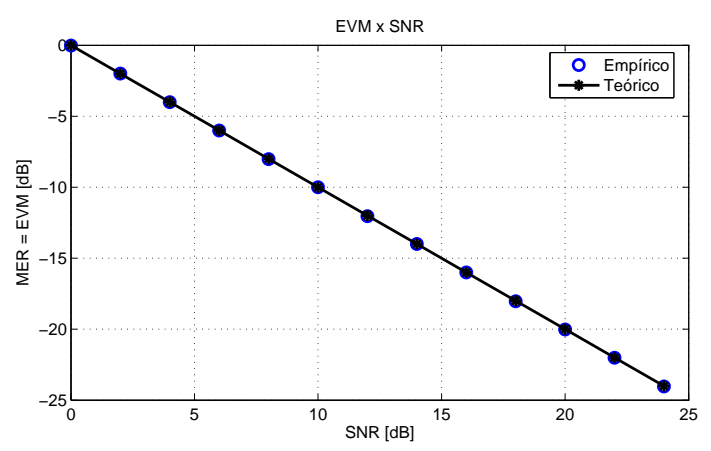

Fig. 5. EVM versus MER do mesmo sistema OFDM da Figura 4.

A exatidão das curvas de simulação computacional (Empírica) e Teórica na avaliação do desempenho do sistema em termos de EVM ou MER por SNR sugere a adoção da relação (16) como parâmetro EVM a ser especificado na equação (15).

\section{AnÁlise de Desempenho}

Para avaliar o desempenho, na relação EVM por SNR, da transmissão de sinais OFDM sob o efeito de não linearidades inseridas por amplificadores de potência e pelo ceifamento de pico em canais com ruído aditivo gaussiano, foram realizados simulações computacionais no software de programação em linguagem técnica MATLAB. Em cada simulação, aproximadamente 1000 símbolos OFDM; divididos em frames de 100 sinais, contendo cada um $N=768$ subportadoras de informação mapeadas em 16-QAM; foram transmitidos em um canal AWGN para diversos valores de SNR. Demapeados, os subsímbolos complexos foram comparados com os transmitidos para a computação da EVM conforme equação (16).

\section{A. Degradação devido à Não Linearidades do PA}

Para simular o efeito da não linearidade inserida pelo PA, o parâmetro $I B O=A_{\text {sat }}^{2} / P_{\text {in }}$ (input power backoff) foi designado, sendo $A_{\text {sat }}$ o nível de saturação de entrada do PA e $P_{i n}=E\left\{\left|x_{n}(t)\right|^{2}\right\}=E\left\{A^{2}(t)\right\}$ a potência média do sinal de entrada do amplificador, para $x_{n}(t)=A(t) e^{j \phi(t)}$. Assim, quanto maior for a IBO, menor será o efeito das não linearidades do PA, à luz da ineficiência de operação do mesmo. A Figura 6 ilustra o desempenho do sistema para diversos valores de IBO, $g_{0}=1, p_{\text {sat }}=1$, bem como a curva de referência para canais com ruído AWGN apenas.

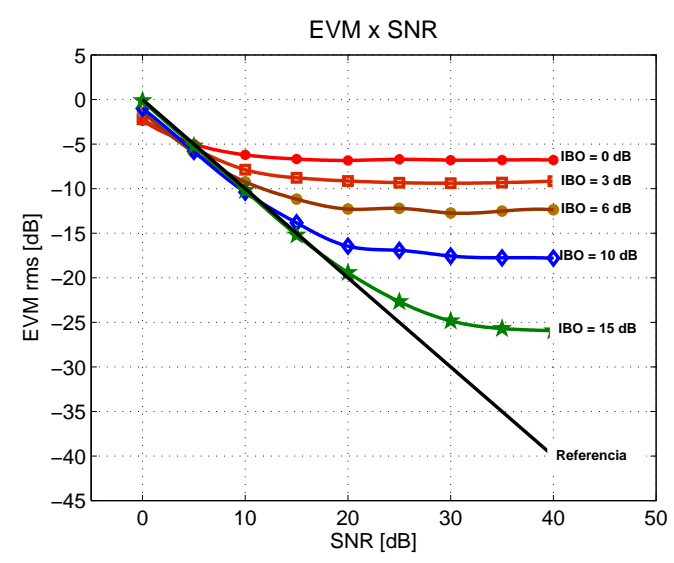

Fig. 6. EVM versus SNR do sistema OFDM sob efeito do PA.

É especificado em [4] um valor de $E V M=-24.4 \mathrm{~dB}$ de referência de desempenho aceitável para sistemas OFDM com mapeamento 16-QAM. Conforme ilustra a Figura 6, apenas a curva referente à $I B O=16 \mathrm{~dB}$ satisfaz esta exigência, para valores de $S N R>27 \mathrm{~dB}$. Isto é perfeitamente explicável pela igualdade $P_{\max }=P_{\text {in }} \cdot P A P R=\frac{P A P R}{I B O} A_{\text {sat }}^{2}$, a qual define que para operação na região linear do PA, a IBO deve ser no mínimo menor que a PAPR, igual a $11 \mathrm{~dB}$ na maioria dos sinais OFDM gerados.

\section{B. Degradação devido às Distorções do Ceifamento de Pico}

Conhecido como um simples e eficiente método de redução do PAPR de sinais OFDM, o clipping introduz distorções em tais sinais na forma de ICI nas subportadoras. Para medir a gravidade deste processo, é definido uma taxa de clipping $\gamma_{c l i p}=A_{\max } / \sqrt{P_{i n}}$, onde $A_{\max }$ é o limiar de corte. A Figura 7 mostra a degradação no desempenho do sistema OFDM simulado para alguns valores de $\gamma_{\text {clip }}$.

Nota-se pela Figura 7 que, quanto maior o $\gamma_{\text {clip }}$ menor é a degradação provocada pelo clipping e maior é o PAPR do sinal após o ceifamento, o que contradiz com o propósito inicial deste processo. Conclui-se portanto, que o processo de ceifamento de pico não é eficiente uma vez que degrada o desempenho do sistema na inserção de distorção aos sinais OFDM, embora reduza o PAPR dos mesmos. Apenas os valores de $\gamma_{\text {clip }} \geq 4 \mathrm{~dB}$ provem níveis de EVM aceitáveis.

\section{Degradação provocada por Desbalanceamento $I / Q$}

Nesta avaliação, apenas a degradação causada pelo desbalanceamento de fase para simular a rotação da constelação 


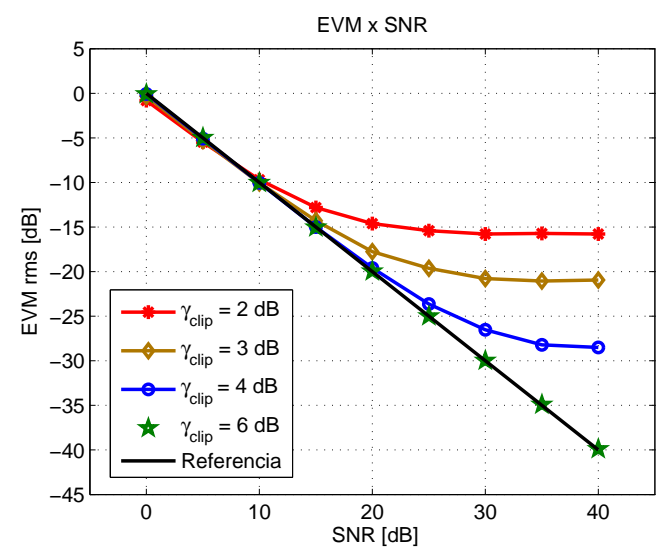

Fig. 7. EVM versus SNR do sistema OFDM sob efeito do clipping.

em sistemas de modulação digital com ruído de fase, foi considerada. Para isso variou-se o valor de $\Delta_{\phi}$ em graus para a avaliação da EVM do sistema em um canal AWGN, conforme ilustra a Figura 8(a). Conforme esperado, à medida em que aumenta-se o desvio na fase dos símbolos OFDM transmitidos aumenta-se drásticamente a EVM dos subsímbolos. Porém, o fato da EVM medir a fase dos subsímbolos transmitidos/recebidos permite corrigir a rotação da constelação gerada por ruídos de fase conforme mostrada na Figura 8(b), onde um desvio de $\Delta_{\phi}=30^{\circ}$ foi perfeitamente corrigido a partir da estimação do desvio de fase inserido.

\section{Conclus Ão}

A adoção da EVM como figura de mérito para a avaliação de desempenho foi apresentado neste artigo em simulações de transmissão de sinais OFDM, com $N=768$ subportadoras mapeadas em 16-QAM, corrompidos com interferências não lineares, através de um canal com ruído AWGN. Enquanto que os efeitos não lineares oriundos de amplificadores de potência mostraram-se toleráveis $(E V M<-24.4 \mathrm{~dB})$ para valores de $I B O \geq 15 \mathrm{~dB}$, os provenientes do ceifamento de pico dos sinais gerados foram permitidos para $\gamma_{\text {clip }} \geq 4 \mathrm{~dB}$, ambos para valores de $S N R \geq 30 \mathrm{~dB}$, onde conclui-se que o clipping é de facto um técnica ineficiente de redução de PAPR. Os resultados de simulação também demonstraram a capacidade da métrica EVM em estimar e corrigir desvios de fase de $\Delta_{\phi}=30^{\circ}$ responsáveis por rotação de diagramas de constelação de sistemas com modulação digital. Aliado à estes recursos intrínsecos, a rapidez de geração e a simplicidade de interpretação de resultados sugerem a adoção da EVM como métrica de avaliação de desempenho de sistemas multiportadoras OFDM, principalmente quando os efeitos de degradação por estes sofridos são não lineares.

\section{REFERÊNCIAS}

[1] L. Hanzo, W. Webb e T. Keller, Single and Multi-carrier Quadrature Amplitude Modulation, John Wiley Sons, 2000.

[2] T. L. Dezan and J. A. L. Silva and K. Côco e E. O. T. Salles and M. E. V. Segatto, Comparação das técnicas de modulação multiportadoras aplicadas às comunicações via rede elétrica, Simpósio Brasileiro de Telecomunicações - SBT'2004, 2004.

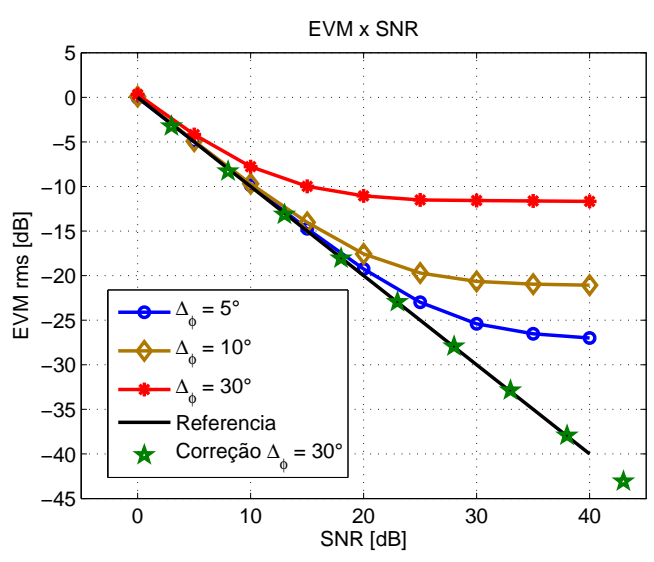

(a)

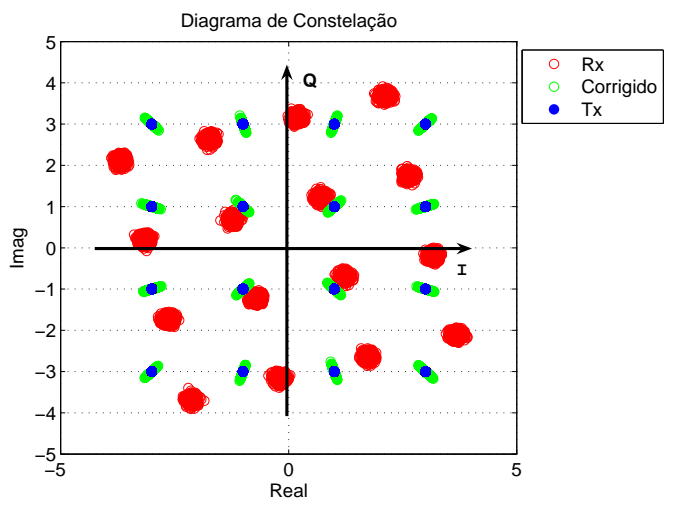

(b)

Fig. 8. Desempenho EVM x SNR do sistema sob efeito do ruído de fase e correção do mesmo via EVM.

[3] J. A. L. Silva, T. M. Siqueira, M. R. N. Ribeiro, R. V. Andreão, E. T. O. Salles e M. E. V. Segatto, A Comparative Analysis of the Performance of OFDM and COFDM Indoor PLC Systems, $13^{\text {th }}$ International Conference on Telecommunications - ICT'06, 2006.

[4] IEEE standart for local and metropolitan area networks part 16: Air interface for fixed broadband wireless access systems , IEEE Std 802.162004, pp. 1-857, 2004.

[5] Shafik, R.A. and Rahman, S. e AHM Razibul Islam, On the Extended Relationships Among EVM, BER and SNR as Performance Metrics, International Conference on Electrical and Computer Engineering ICECE'06, 2006, pp. 408-411.

[6] IEEE standard for wireless LAN Medium Access Control (MAC) and Physical Layer (PHY) specifications: high-speed physical layer in the 5 GHz band, IEEE Std 802.11a-1999, 1999

[7] Chunming Zhao e Baxley, R.J., Error Vector Magnitude Analysis for OFDM Systems, Asilomar Conference on Signals, Systems and Computers - ACSSC '06, 2006, pp. 1830-1834.

[8] M. D. McKinley, K. A. Remley, M. Mylinski, J. S. Kenney, D. Schreurs, e B. Nauwelaers, EVM Calculation for Broadband Modulated Signals, Technical report of United States Goverment, 2005.

[9] S. Yamanouchi, K. Kunihiro e H.Hida, OFDM Error Vector Magnitude Distortion Analysis, IEICE Transactions, 2006, vol 89-C, pp. 1836-1842.

[10] V.J. Urick, J.X. Qiu e F. Bucholtz, Wide-band QAM-over-fiber using phase modulation and interferometric demodulation, IEEE Photonics Technology Letters, 2004, vol 16, pp. 2374-2376. 